\title{
The novel resveratrol analog HS-1793-induced polyploid LNCaP prostate cancer cells are vulnerable to downregulation of $\mathrm{Bcl}-\mathrm{xL}$
}

\author{
NA YOUNG JEONG ${ }^{1}$, YOUNG GEOL YOON ${ }^{1}$, JEE HYUN RHO $^{1}$, JEE SUK LEE ${ }^{1}$, SANG YEOB LEE $^{1}$, \\ KI SOO YOO ${ }^{1}$, SUHEE SONG ${ }^{2}$, HONGSUK SUH ${ }^{2}$, YUNG HYUN CHOI ${ }^{3}$ and YOUNG HYUN YOO ${ }^{1}$
}

\author{
${ }^{1}$ Department of Anatomy and Cell Biology and Mitochondria Hub Regulation Center, Dong-A University College of \\ Medicine; ${ }^{2}$ Department of Chemistry and Chemistry Institute for Functional Materials, Pusan National University; \\ ${ }^{3}$ Department of Biomaterial Control (BK21 Program), Dongeui University Graduate School, Busan, Republic of Korea
}

Received January 17, 2011; Accepted February 28, 2011

DOI: $10.3892 /$ ijo.2011.979

\begin{abstract}
Since resveratrol is not a potent cytotoxic compound when compared with other chemotherapeutic agents, several previous studies have been performed to obtain synthetic analogs of resveratrol with potent activity. Our previous study demonstrated that the resveratrol analog HS-1793 showed stronger antitumor activity than resveratrol in various cancer cells. We examined the antitumor activity exerted by HS-1793 in prostate cancer cells, and we observed that HS-1793 acts as a polyploidy inducer. Noticeably, multinucleation and polyploidization were induced in most $\mathrm{LNCaP}$ cells treated with HS-1793 at the dose causing a slight decline in cell viability. However, the induction of multinucleation and polyploidization was much lower in $\mathrm{PC}-3$ prostate cancer cells treated with the same dose of HS-1793. Western blot and RT-PCR analyses showed that the expression of Aurora B was almost undetectable in $\mathrm{LNCaP}$ cells, but it was highly expressed in PC-3 cells. Further, silencing of Aurora B sensitized PC-3 cells to HS-1793-induced multi-nucleation. These results indicate that expression of Aurora B determines multinucleation in prostate cancer cells treated with HS-1793. Additional assays using multiple cancer cell lines show that the population of multinucleated cells induced by HS-1793 treatment is inversely proportional to Aurora B expression. We further elicited that the HS-1793-induced polyploid LNCaP cells are vulnerable to downregulation of Bcl-xL. Since the polyploidization in LNCaP induced by HS-1793 does not appear to cause definite commitment to apoptosis, the termination of polyploid cells by inhibition of Bcl-xL could provide an advantageous means to improve chemotherapeutic efficacy of HS-1793.
\end{abstract}

Correspondence to: Dr Young Hyun Yoo, Department of Anatomy and Cell Biology, Dong-A University College of Medicine and Mitochondria Hub Regulation Center, 3-1 Dongdaesin-dong, Seo-gu, Busan 602-714, Republic of Korea

E-mail: yhyoo@dau.ac.kr

Key words: resveratrol analog, multinucleation, polyploidy, Aurora B kinase, Bcl-xL

\section{Introduction}

The polyphenolic compound resveratrol (3,5,4-trihydroxytrans-stilbene) is a naturally occurring phytochemical found in food products such as grapes, peanuts, and various herbs. Resveratrol acts on the process of carcinogenesis by affecting cellular events associated with tumor initiation, promotion, and progression (1). Resveratrol is also able to induce apoptosis (2). The chemopreventative and chemotherapeutic properties associated with resveratrol offer promise for the design of new chemotherapeutic drugs. However, resveratrol is not a potent cytotoxic compound when compared with other chemotherapeutic agents. Therefore, exposure to high doses of resveratrol is required to exert antitumor efficacy (3). Furthermore, the biological activity of resveratrol is limited by its photosensitivity and metabolic instability (4). Accordingly, several previous studies have been performed to test synthetic analogs of resveratrol with potent activity.

Our previous study, using four resveratrol analogs designed and synthesized by us (5), demonstrated that those analogs showed stronger antitumor activity than resveratrol in various cancer cells (6). We elucidated that the antitumor activity of a resveratrol derivative 4-(6-hydroxy-2-naphthyl)-1,3-benzenediol (HS-1793) is mediated by the downregulation of 14-3-3 in human leukemic U937 cells.

Prostate cancer $(\mathrm{CaP})$ develops in the tissues of the prostate gland in the male reproductive system. It is one of the most common cancers in men and is the second leading cause of male cancer mortality worldwide after lung cancer (7). Previous studies have reported that resveratrol, a naturally occurring phytoalexin found in red wine, grapes, peanuts, and a variety of medicinal plants, has the ability to inhibit the carcinogenic process at initiation, promotion, and progression and induce apoptosis in various tumor cells, including prostate cancer cells $(1,8-10)$. It has been reported that resveratrol inhibits $\mathrm{CaP}$ cell proliferation and suppresses the expression of prostate specific genes such as prostate-specific antigen (PSA) and the androgen receptor (AR) (11). In addition, several potential targets of resveratrol have been reported, including NF- $\mathrm{kB}, \mathrm{AP}-1, \mathrm{p} 53, \mathrm{Bcl}-2$ and Bax, but the mechanism by which resveratrol inhibits cancer cell growth is still not fully understood 
$(8,10)$. The antitumor effects of resveratrol analogs on prostate cancers have also been studied $(8,10)$.

Aurora kinases are serine/threonine protein kinases that regulate several processes during cell division such as chromosome alignment, segregation, and cytokinesis. Mammalian cells express three Aurora kinases: Aurora A, B and C (12). The three Aurora kinases are highly similar in sequence and structure. However, the three mammalian Aurora kinases are known to be different in their subcellular distribution and functions (13). Aurora A localizes to the spindle poles where it plays an important role in centrosome separation, mitotic entry, and spindle assembly (14). Aurora B is a key regulator of chromosome alignment, microtubule-kinetochore attachment, spindle check point, and cytokinesis $(13,14)$. Less is known about Aurora C, which is specifically expressed at high levels in the testes and localizes to centrosomes from anaphase to telophase (15).

Significant overexpression of Aurora B has been shown in a wide range of human cancer cells (16). In addition, elevated levels of Aurora kinases correlate with advanced clinical stages of cancers (17). Therefore, Aurora kinases have become propitious targets for cancer therapy and are being evaluated in clinical trials for the treatment of cancer. Several small molecule inhibitors of Aurora kinases have been developed including ZM447439 (Astra Zeneca), Hesperadin (Boehringer Mannheim), VX-680 (Vertex Inc.), and AZD1152 (17,18). The typical cellular phenotype induced by the inhibition of Aurora B is a failure of normal cytokinesis with the induction of polyploidy and multinucleated giant cells (19). Pharmacological incapacitation of the spindle assembly checkpoint with small-molecule Aurora B inhibitors induces apoptosis secondary to polyploidization $(18,20)$. Thus, Aurora B inhibitors are being evaluated in clinical trials for the treatment of cancer. Aurora kinase expression appears to influence prostate cell proliferation (17), and Aurora kinase expression directly correlates with prostate cancer malignancy $(17,21)$. While Aurora B is highly expressed in PC-3 prostate cancer cells, its expression in $\mathrm{LNCaP}$ prostate cancer cells is much weaker than in PC-3 cells or almost undetectable (17).

We performed this study to examine the mechanism underlying the antitumor activity of HS-1793 in prostate cancer cells. HS-1793 induces the formation of multinucleated polyploid cells in LNCaP human prostate cancer cells. Further, HS-1793-induced polyploid LNCaP prostate cancer cells are vulnerable to downregulation of Bcl-xL.

\section{Materials and methods}

Reagents. The following reagents were obtained commercially: rabbit polyclonal anti-human Bcl-2, Mcl-1, and Bcl-xL antibodies and ABT-263 from Santa Cruz Biotechnology (Santa Cruz, CA); rabbit polyclonal anti-human caspase-3 and p53 antibodies from Cell Signaling Technology (Danvers, MA); HRP-conjugated donkey anti-rabbit and sheep anti-mouse IgG antibodies from Amersham Pharmacia Biotech (Piscataway, NJ); $\beta$-actin antibody, Hoechst 33342, dimethylsulfoxide (DMSO), RNase A, proteinase K, leupeptin, propidium iodide (PI), and Giemsa solution from Sigma (St. Louis, MO); rabbit monoclonal anti-human Aurora B from Epitomics (Burlingame, CA); neomycine sulfate (G418) from Calbiochem (San Diego, CA); RPMI-1640 and fetal bovine serum (FBS) from Gibco
(Gaithersburg, MD); and Super Signal West Pico enhanced chemiluminescence Western blotting detection reagent from Pierce (Rockford, IL).

Preparation of resveratrol analogs. HS-1793 was prepared as described previously (6).

Cell culture. Human prostate cancer cells PC-3 and LNCaP and other cancer cells K562, U937, HCT116 and T98G were obtained from the American Type Culture Collection (ATCC, Rockville, MD). ZR-75-1 and U373MG were purchased from the Korean Cell Line Bank (KCLB, Seoul, Korea). The anaplastic thyroid cancer cell line KAT-18 was kindly provided by Dr K.B. Ain (University of Kentucky, Chandler Medical Center, Lexington, KY). The culture medium used throughout these experiments was RPMI-1640 medium with $10 \%$ fetal calf serum (FCS), $20 \mathrm{mM}$ HEPES buffer and $100 \mu \mathrm{g} / \mathrm{ml}$ gentamicin.

Resveratrol analog treatment and assessment of cell viability. Resveratrol analogs $(10 \mu \mathrm{M})$ in ethanol were prepared and stored at $-80^{\circ} \mathrm{C}$ until use. Cells were treated with resveratrol analogs (up to $40 \mu \mathrm{M}$ ). Cells were harvested $48 \mathrm{~h}$ after treatment, and cell viability was determined with the Vi-Cell cell counter (Beckman Counter, Fullerton, CA), which performs an automated trypan blue exclusion assay.

Nuclear morphology analysis of apoptosis. The cell suspension was cytospun onto clean fat-free glass slides with a cytocentrifuge. Cytocentrifuged samples were fixed for $10 \mathrm{~min}$ in $4 \%$ paraformaldehyde solution and stained in $4 \mu \mathrm{g} / \mathrm{ml}$ of Hoechst 33342 for $30 \mathrm{~min}$ at $4^{\circ} \mathrm{C}$. The cells were observed and photographed under an epifluorescence microscope. To quantify cells showing multinucleation, the total cell number (300 cells from each experiment) was counted using differential interference contrast (DIC) optics by an observer who was blinded to the experimental group.

Reverse transcriptase (RT)-PCR. Total cellular RNA was isolated from different cell lines using the RNeasy Mini kit (Qiagen, GmbH, Germany). Total RNA ( $2 \mu \mathrm{g})$ was reversetranscribed using oligo-dT primers and M-MLV reverse transcriptase. Approximately 1 to $3 \mu \mathrm{l}$ of reverse transcription-PCR reactions were subjected to 30 cycles of PCR for amplification of Aurora B and $\beta$-actin. The PCR was performed in a $50 \mu 1$ reaction volume containing $1 \mu \mathrm{M}$ primers, $200 \mu \mathrm{M}$ of each dNTPs and 1.25 units of Taq DNA polymerase. After denaturing at $95^{\circ} \mathrm{C}$ for $2 \mathrm{~min}$, cDNA was subjected to 30 cycles of PCR amplification. The PCR conditions were $95^{\circ} \mathrm{C}$ for $40 \mathrm{sec}, 60^{\circ} \mathrm{C}$ for $40 \mathrm{sec}$ and $72^{\circ} \mathrm{C}$ for $100 \mathrm{sec}$ for Aurora $\mathrm{B}$ and $\beta$-actin amplification with a final extension of $72^{\circ} \mathrm{C}$ for $5 \mathrm{~min}$. The PCR-amplified products were electrophoresed in a $1 \%$ or $1.5 \%$ agarose gel and stained with ethidium bromide. Expression was standardized using the $\beta$-actin signal as an internal control. The sequence of oligonucleotide primers is as follows: Aurora B-forward primer, 5'-CGACATCTTAACGCGGCAC-3'; Aurora B-reverse primer, 5'-GGACGCCCAATCTCAAAGTC-3' (50 bp fragments); $\beta$-actin-forward primer, 5'-TGACGGGGTCACCCACACT GTGCCCAT-3'; and $\beta$-actin-reverse primer, 5'-CTAGAAG CATTTGCGGTGGACGATGGAGGG-3' (660 bp fragments). 
A

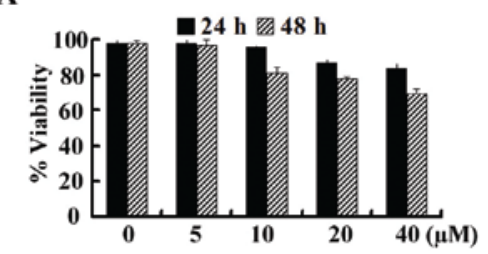

B

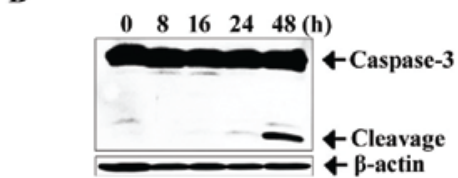

C
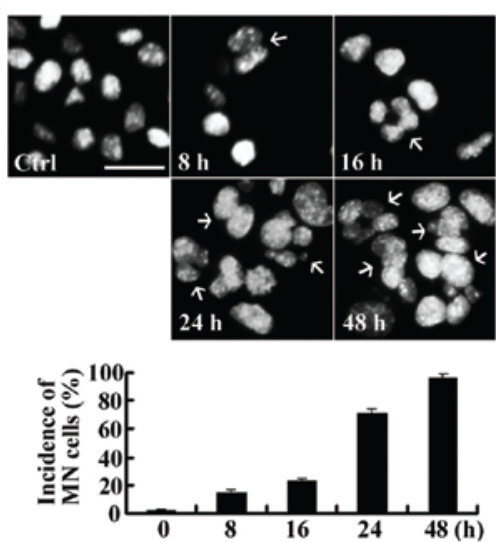

D

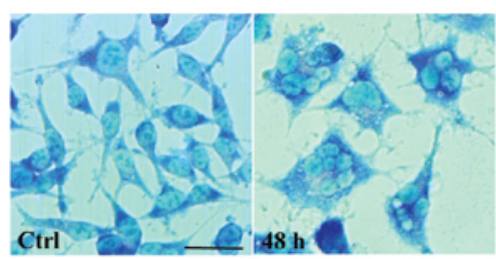

E

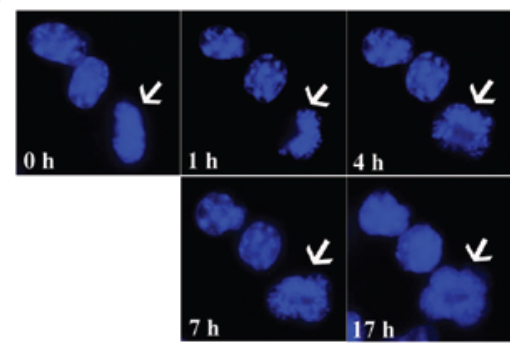

G

$\begin{array}{llllll}0 & 8 & 16 & 24 & 48 & \text { (h) }\end{array}$
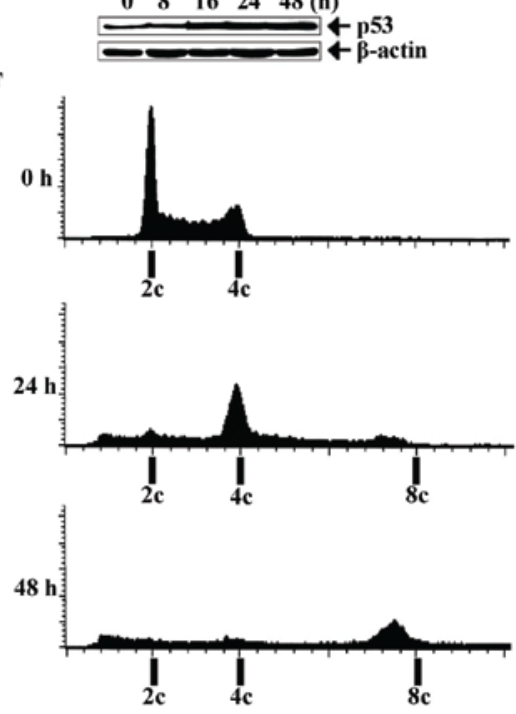

Figure 1. HS-1793 induces multinucleation and polyploidization in LNCaP human prostate cancer cells. (A) Viability assay after treating with HS-1793 for $24 \mathrm{~h}$ (solid) and $48 \mathrm{~h}$ (hatched). Viability was determined by automated trypan blue exclusion assay with a cell counter. HS-1793 produced a dose-dependent reduction in LNCaP cell viability. Based on the viability assay, cells were incubated with $10 \mu \mathrm{M}$ of HS-1793 (B-G). (B) Western blot assay. HS-1793 produced the cleaved product of caspase-3. $\beta$-actin expression was determined to confirm equal protein loading. (C) Hoechst staining. Multinucleated cells were observed in HS-1793 treated cells (upper panel, see arrows). The percentage of multinucleated cells is depicted in a diagram (lower panel). The data represent the mean \pm SD of at least four independent experiments. MN, multinucleated. (D) Giemsa staining. Most of the HS-1793-treated cells have more than two nuclei. (E) Live cell imaging. Representative frames from the time-lapse video are shown. The time stamp indicates hours after the addition of HS-1793. The increase in the number of nuclei is a continual process (see arrows). (F) Flow cytometric analysis. The $\mathrm{G}_{1}$ DNA content of $2 \mathrm{c}$, the $\mathrm{G}_{2}$ DNA content of $4 \mathrm{c}$ and the second $\mathrm{G}_{2}(+)$ DNA content of $8 \mathrm{c}$ are indicated under the $\mathrm{X}$ axes of the histograms. Representative histograms showing the induction of polyploid cells having $8 \mathrm{c}$ after HS-1793 treatment are presented. (G) Western blot assay. HS-1793 increased the expression of p53 protein. $\beta$-actin expression was determined to confirm equal protein loading. Bar, $50 \mu \mathrm{m}$.

Flow cytometric analysis. Ice-cold $95 \%$ ethanol supplemented with $0.5 \%$ Tween-20 was added to cell suspensions to a final concentration of $70 \%$ ethanol. Fixed cells were pelleted and washed in $1 \%$ BSA-PBS solution. The cells were re-suspended in $1 \mathrm{ml}$ PBS containing 11 Kunitz $\mathrm{U} / \mathrm{ml}$ RNase, incubated at $4^{\circ} \mathrm{C}$ for $30 \mathrm{~min}$, washed once with BSA-PBS, and re-suspended in a PI solution $(50 \mu \mathrm{g} / \mathrm{ml})$. The cells were incubated at $4^{\circ} \mathrm{C}$ for $30 \mathrm{~min}$ in the dark and were then filtered through $35 \mathrm{~mm}$ mesh, and the DNA content was determined using a FACSCalibur (Becto-Dickinson) flow cytometer within $1 \mathrm{~h}$. The cellular DNA content was analyzed by CellQuest software (BectonDickinson).

Western blot analysis. Cell lysates were centrifuged at $14,000 \mathrm{rpm}$ for $15 \mathrm{~min}$ at $4^{\circ} \mathrm{C}$. The protein concentrations of the cell lysates were determined using a Bradford protein assay
(Bio-Rad), and equal amounts of proteins were loaded onto 7.5-15\% SDS-polyacrylamide gels. The gels were transferred to nitrocellulose membranes (Amersham Pharmacia Biotech, Piscataway, NJ) and blotted with each antibody. Immunostaining with antibodies was performed using Super Signal West Pico enhanced chemiluminescence substrate and detected with LAS-3000PLUS (Fuji Photo Film Company, Kanagawa, Japan).

siRNA transfection. To determine the effect of Aurora B knockdown, the following double-stranded RNA oligonucleotides were used: sense: 5'-AAGGAGAACUCCUACCC CUGGUU-3', antisense: 5'-CCAGGGGUAGGAGUUCUCU UUU-3'. For Bcl-2, Mcl-1, and Bcl-xL depletion, pre-designed siRNA constructs were purchased. As a negative control, the same nucleotides were scrambled to form non-targeting combinations. All of the siRNA duplexes were synthesized by 
A

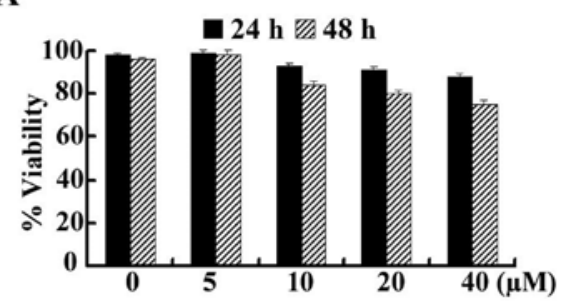

B

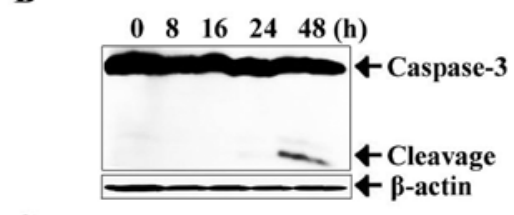

C
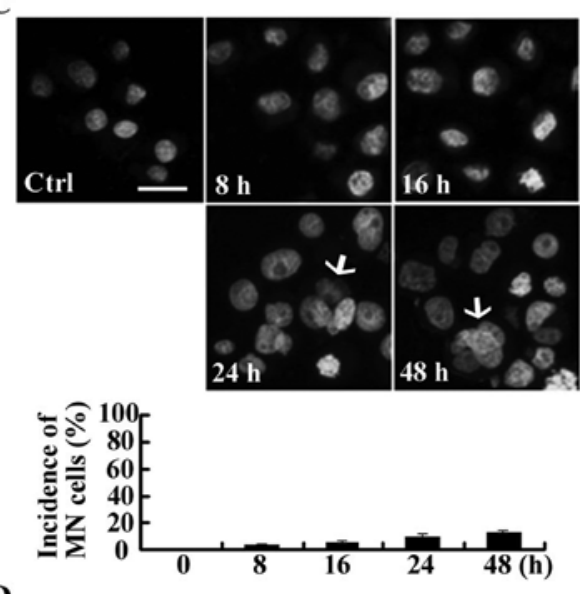

D
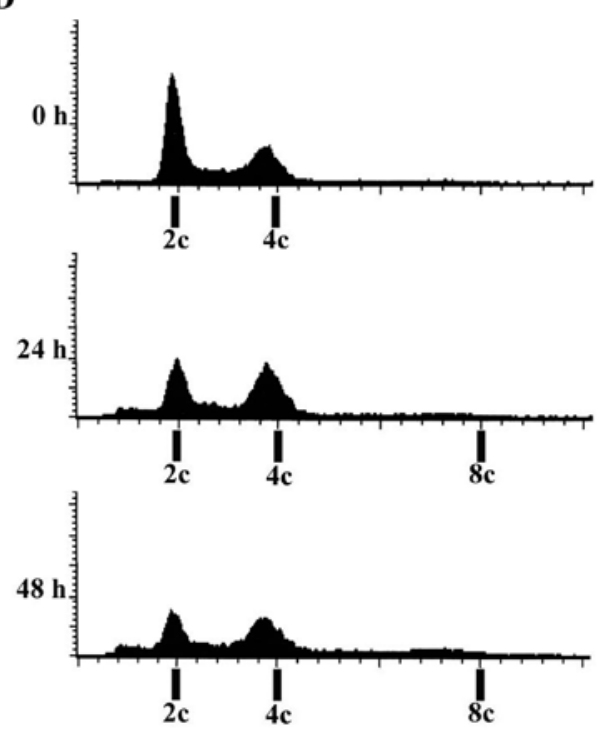

Figure 2. Induction of multinucleation and polyploidization by treatment with HS-1793 in PC-3 cells was less significant than in LNCaP cells. (A) Viability assay $24 \mathrm{~h}$ (solid) and $48 \mathrm{~h}$ (hatched) after HS-1793 treatment. HS-1793 produced a dose-dependent reduction in PC3 cell viability. (B) Western blot assay. HS-1793 induced an increase in the cleaved product of caspase-3. $\beta$-actin expression was determined to confirm equal protein loading. (C) Hoechst staining. Multinucleated cells were observed in HS-1793 treated cells (upper panel, see arrows). The percentage of multinucleated cells was depicted in a diagram (lower panel). The data represent the mean \pm SD of at least four independent experiments. Fewer multinucleated cells were observed in PC-3 cells compared to those in LNCaP cells. Bar, $50 \mu \mathrm{m}$. (D) Flow cytometric analysis. The $\mathrm{G}_{1}$ DNA content of $2 \mathrm{c}$, the $\mathrm{G}_{2}$ DNA content of $4 \mathrm{c}$ and the second $\mathrm{G}_{2}(+)$ DNA content of $8 \mathrm{c}$ are indicated under the $\mathrm{X}$ axes of the histograms. Representative histograms are presented. Polyploid cells having $8 \mathrm{c}$ in PC-3 cells are very few compared to those in LNCaP cells. Bar, $50 \mu \mathrm{m}$. See Fig. 1 for other definitions.
Dharmacon (Chicago, IL) and were transfected using siPORT Amine (Ambion, TX) in OptiMEM (Bio Sciences, Dublin, Ireland) according to the manufacturer's recommendations.

Live cell imaging. Live LNCaP cells were plated on $35 \mathrm{~mm}$ dishes $24 \mathrm{~h}$ prior to treatment. Hoechst 33342 solution was added in the tissue culture media at $3 \mu \mathrm{g} / \mathrm{ml}$ for $30 \mathrm{~min}$. The cells were then washed and cultured with HS-1793 at $10 \mu \mathrm{M}$. Live cell observation was performed in a temperature controlled chamber for $17 \mathrm{~h}$ using an integrated Delta Vision system (Applied Precision, Issaquah, WA). For monitoring multinucleation, at least 5 fields containing 3 to 7 cells were observed.

Giemsa staining. LNCaP cells were cultured without or with HS-1793 and then fixed with 3:1 methanol/acetic acid for $10 \mathrm{~min}$. For polyploidy observation, slides were stained for $10 \mathrm{~min}$ with Giemsa solution.

Statistical analysis. Four independent in vitro experiments were carried out. Statistical results were expressed as the mean \pm SD of the means obtained from triplicates of each independent experiment. Statistical significance of differences was determined by the paired Kruskal-Wallis non-parametric test. $\mathrm{P}<0.05$ was considered significant.

\section{Results}

HS-1793 induces multinucleation and polyploidization in LNCaP human prostate cancer cells. As determined by trypan blue assay, HS-1793 reduced the viability of human prostate cancer LNCaP cells in a dose-dependent manner. To further study the mechanism of the cytotoxicity induced by HS-1793, LNCaP cells were cultured in the absence or presence of HS-1793 at $10 \mu \mathrm{M}$, which is the lowest dose that caused a decline (approximately 20\%) in cell viability $48 \mathrm{~h}$ after treatment. Western blot assay showed degradation of caspase-3, indicating that HS-1793 reduced the viability of human prostate cancer LNCaP cells via the caspase-mediated pathway (Fig. 1B). Noticeably,HS-1793 induced multinucleation in LNCaP cells. Forty eight hours after treatment with HS-1793, most LNCaP cells were multinucleated (Fig. 1C and D). Using live cell imaging, we were able to directly observe the formation of a multinucleated cell after treatment with HS-1793. The increase in the number of nuclei was a continuous process (Fig. 1E). Flow cytometry demonstrated that $\mathrm{LNCaP}$ cells became fully octaploid (8c) by $48 \mathrm{~h}$, as shown by an $8 \mathrm{c}$ DNA peak (Fig. 1F). We further examined the expression level of p53 protein, which is a substitute cellular marker of polyploidization in cells with wild-type p53 genetic status. As expected, the expression level of p53 was substantially increased in HS-1793-treated polyploid LNCaP cells (Fig. 1G).

HS-1793 did not substantially induce multinucleation in PC-3 human prostate cancer cells. We next examined whether HS-1793 also induced multinucleation in PC-3 human prostate cancer cells. As determined by trypan blue assay, HS-1793 also reduced the viability of human prostate cancer PC-3 cells in a dose-dependent manner. HS-1793 at $10 \mu \mathrm{M}$ reduced the viability by approximately $20 \%$ concomitant with the degradation of 
$\mathbf{A}$

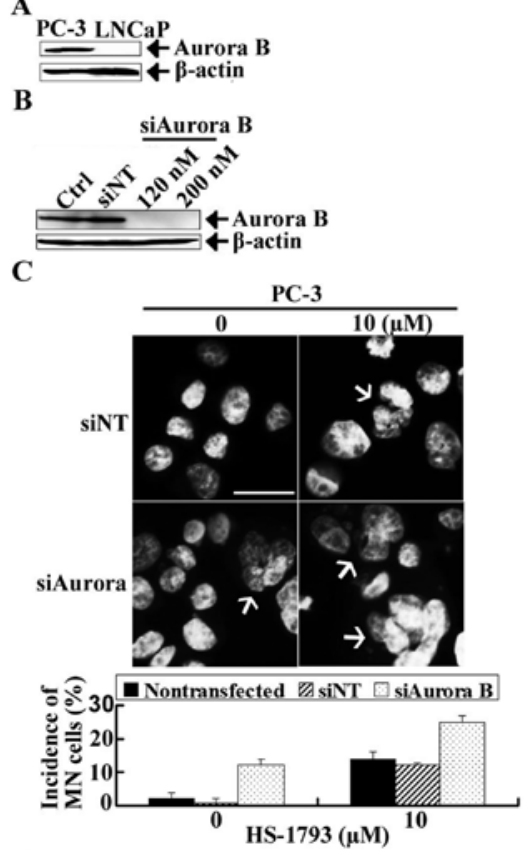

D
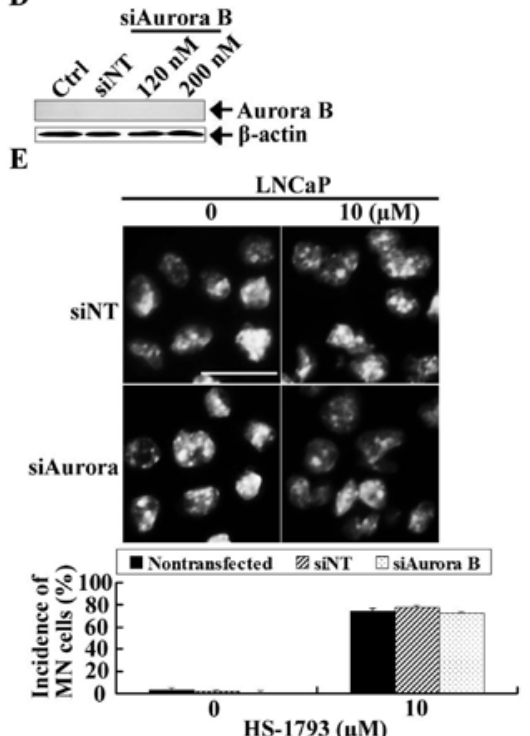

Figure 3. Expression levels of Aurora B determine the multinucleation in prostate cancer cells treated with HS-1793. Ctrl, non-transfected control; siNT, non-target control siRNA. (A) Western blot assay reveals that the expression level of Aurora B kinase in PC-3 cells is high, while it is almost undetectable in LNCaP cells. (B) Western blot assay: both 120 and $200 \mathrm{nM}$ of siRNA against Aurora B (siAurora B) efficiently depleted Aurora B kinase in PC-3 cells. (C) Hoechst staining. After silencing Aurora B by siRNA, PC-3 cells were incubated for $48 \mathrm{~h}$ with $(10 \mu \mathrm{M})$ or without HS-1793. Many multinucleated cells are observed in Aurora B-silenced PC-3 cells compared to the experimental controls (upper panel, see arrows). The percentages of multinucleated cells are depicted in a diagram (lower panel). Solid, non-transfected control; hatched, transfected with non-target control siRNA; dotted, transfected with Aurora B siRNA. The population of multinucleated cells was significantly increased in PC-3 cells transfected with Aurora B siRNA compared to those in the non-transfected control and the experimental control transfected with non-targeting siRNA $\left({ }^{* *} \mathrm{P}<0.01\right)$. (D) Western blot assay. Aurora B was not detected in all groups of LNCaP cells. (E) Hoechst staining. LNCaP cells were incubated for $24 \mathrm{~h}$ with $(10 \mu \mathrm{M})$ or without HS-1793 after Aurora B silencing (upper panel). The percentages of multinucleated cells are depicted in a diagram (lower panel). Aurora B silencing in LNCaP cells did not alter the population of multinucleated cells. Solid, non-transfected control; hatched, transfected with non-target control siRNA; dotted, transfected with Aurora B siRNA. There is no significant difference in the population of multinucleated cells among these three groups $(\mathrm{P}>0.05)$. Bar, $50 \mu \mathrm{m}$. See Fig. 1 for other definitions caspase-3 (Fig. 2B). Intriguingly, the induction of multinucleation and polyploidization by HS-1793 was much lower in PC-3 cells than in LNCaP cells. Morphological observation showed that HS-1793 did not substantially increase the population of multinucleated cells in PC-3 cells. The percent of multinucleated cells $48 \mathrm{~h}$ after treatment was less than $10 \%$ (Fig. 2C). Flow cytometry also demonstrated that HS-1793 did not substantially increase the population of polyploid cells (Fig. 2D). The percent of polyploid cells $48 \mathrm{~h}$ after treatment was less than $10 \%$.

Expression of Aurora $B$ determines multinucleation in prostate cancer cells treated with HS-1793. We next examined whether the expression of Aurora B determines multinucleation in prostate cancer cells treated with HS-1793. Western blot analysis showed that the expression of Aurora B is almost undetectable in LNCaP cells, while it is highly expressed in PC-3 cells (Fig. 3A). We next examined whether knock-down of Aurora B sensitized PC-3 human prostate cancer cells to the induction of multinucleation by HS-1793. The introduction of siRNA against Aurora B efficiently depleted Aurora B in PC-3 cells (Fig. 3A). Importantly, siRNA against Aurora B significantly increased the population of multinucleated cells in PC-3 cells treated with HS-1793 compared to the control and the experimental control (Fig. 3C). The effect of Aurora B siRNA on the population of multinucleated cells in LNCaP cells was examined $24 \mathrm{~h}$ after HS-1793 treatment. Treatment with siRNA against Aurora B did not alter the population of multinucleated cell in LNCaP cells (Fig. 3D and E).

The population of multinucleated cells after HS-1793 treatment is inversely proportional to Aurora B expression. To further address the relationship between the incidence of multinucleated polyploid cells induced by HS-1793 and the expression of Aurora B, reverse transcriptase (RT)-PCR was performed with gene-specific primers for Aurora B. Our RT-PCR analysis demonstrated that unlike seven cancer cell lines showing high expression of Aurora B mRNA, LNCaP and T98G cancer cells showed much lower expression of Aurora B mRNA (Fig. 4A). Western blot assay showed that the expression of Aurora B protein was also much lower in T98G cells compared to seven other cancer cells (Fig. 4B). The expression of Aurora B mRNA and protein was almost undetectable in LNCaP cells. Importantly, the number of multinucleated cells induced by HS-1793 treatment was significantly higher in T98G cells compared to seven other cancer cell lines with high expression of Aurora B (Fig. 4C). However, the population of multinucleated cells in T98G treated with HS-1793 was significantly lower than in HS-1793-treated Aurora B-depleted LNCaP cells. These findings indicate that the population of multinucleated polyploid cells induced by HS-1793 treatment is inversely proportional to Aurora B expression.

HS-1793 and inhibition of Bcl-xL elicit synergistic cytotoxicity. Because recent studies have demonstrated that the inhibition of Bcl-xL triggers accelerated death of polyploid cancer cells (22), we further examined whether combined treatment of HS-1793 with ABT-263, which inhibits Bcl-2 family members including Bcl-xL, could trigger significant cytotoxicity. While $1 \mu \mathrm{M}$ ABT-263 treatment alone did not reduce the viability of 
A

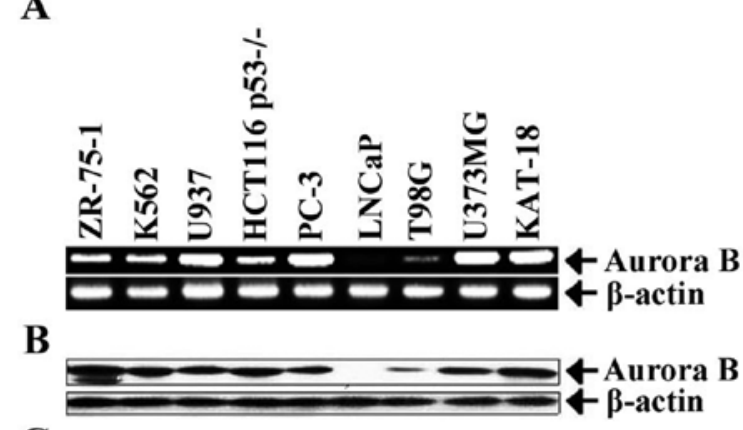

C

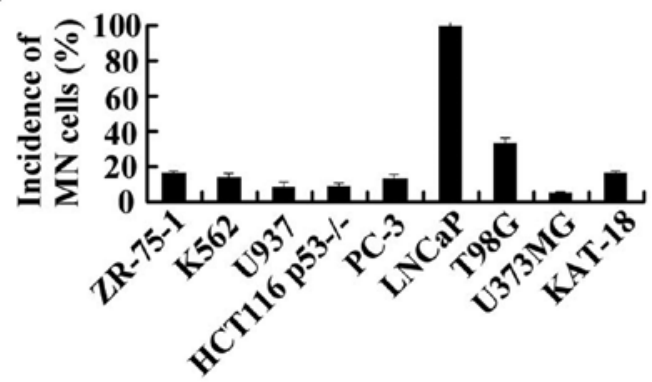

Figure 4. The population of multinucleated cells with HS-1793 treatment is inversely proportional to Aurora B expression. (A) Expression of Aurora B in untreated cells. Semiquantitative RT-PCR was performed to assay Aurora B mRNA expression. A representative result out of four independent experiments is shown. $\beta$-actin was used as an internal control. (B) Western blot analysis showing the expression level of Aurora B protein in untreated cells. $\beta$-actin expression was determined to confirm equal protein loading. (C) The percentage of multinucleated cells in various cancer cells treated with $10 \mu \mathrm{M}$ HS-1793 for $48 \mathrm{~h}$. The number of multinucleated cells induced by HS-1793 is significantly increased in Aurora B-depleted LNCaP cells compared to that of other eight cancer cells $(\mathrm{P}<0.01)$. Further, the number of multinucleated cells induced by HS-1793 is significantly higher in T98G cells weakly expressing Aurora B compared to seven other cancer cells that highly express Aurora B $(\mathrm{P}<0.01)$. See Fig. 1 for other definitions.

LNCaP cells, $1 \mu \mathrm{M}$ ABT-263 in combination with $10 \mu \mathrm{M}$ HS-1793 augmented the death induced by HS-1793. Further, the combination treatment produced cleaved caspase- 3 at earlier time points compared to HS-1793 alone (Fig. 1B). Because ABT-263 inhibits several members of the Bcl-2 family, we next used siRNA to determine which of the ABT-263 targets, when inhibited, would be responsible for the synergistic cytotoxicity exerted by ABT-263 in combination with HS-1793. siRNAs against Bcl-2, Mcl-1 and Bcl-xL efficiently downregulated the expression of their target proteins in LNCaP cells. Silencing of Bcl-2 or Mcl-1 slightly reduced the viability of LNCaP cells regardless of HS-1793 treatment. Importantly, although siRNA against Bcl-xL decreased the viability of untreated LNCaP cells, it significantly reduced the viability of $\mathrm{LNCaP}$ cells treated with HS-1793 compared with siRNA against Bcl-2 or Mcl-1, indicating that synergistic activity of HS-1793 and ABT-263 is mainly due to the inhibition of Bcl-xL (Fig. 5C).

\section{Discussion}

Polyploidy emerges from various defects in DNA replication, dissolving sister-chromatid cohesion, mitotic spindle function or cytokinesis (23-26). Polyploid cells are formed during normal development and in several pathological conditions
A

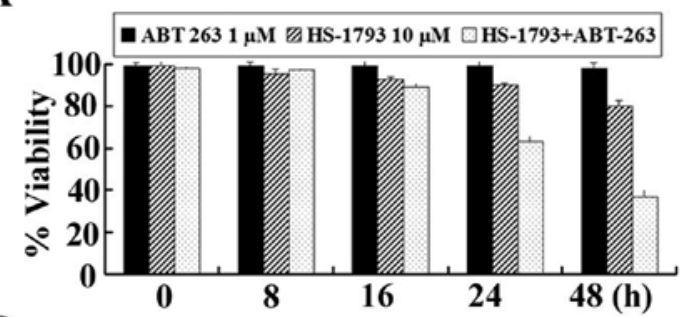

B

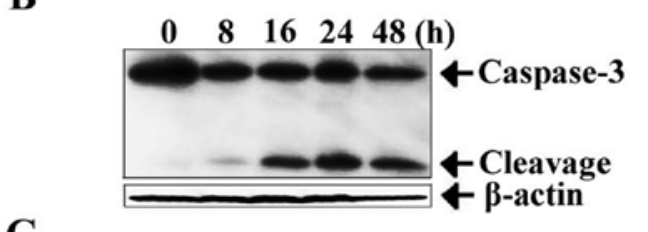

C

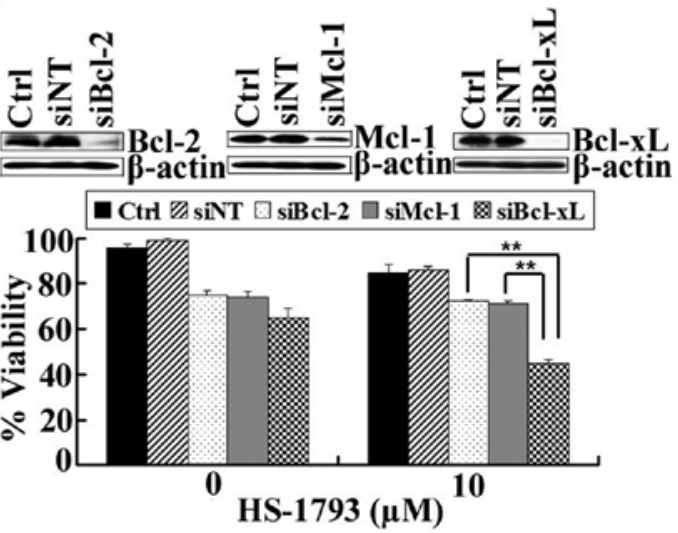

Figure 5. HS-1793 treatment and inhibition of Bcl-xL elicit synergistic cytotoxicity. LNCaP cells were treated with $10 \mu \mathrm{M}$ HS-1793 alone or with combinations of $10 \mu \mathrm{M}$ HS-1793 and $1 \mu \mathrm{M}$ ABT-263. Viability was determined by automated trypan blue exclusion assay with a cell counter. (A) Viability assay. Solid, ABT-263 treatment alone; hatched, HS-1793 treatment alone; dotted, combination treatment with $10 \mu \mathrm{M}$ HS-1793 and $1 \mu \mathrm{M}$ ABT-263. HS-1793 caused synergistic cytotoxicity in combination with ABT-263. (B) Western blot assay showing the production of cleaved caspase- 3 product in cells co-treated with HS-1793 and ABT-263 at the earlier time points compared to cells treated with HS-1793 alone (see Fig. 1B). (C) Effect of siRNAs against Bcl-2, Mcl-1 and Bcl-xL genes. Ctrl, non-transfected control; siNT, non-target control siRNA. SiRNAs against the Bcl-2, Mcl-1 and Bcl-xL genes efficiently downregulated the expression of Bcl-2, Mcl-1 and $\mathrm{Bcl}-\mathrm{xL}$ in LNCaP cells, respectively (upper panels). Viability assay is shown in lower panel. Solid, non-transfected control; hatched, cells transfected with a nontarget control siRNA; dotted, cells transfected with Bcl-2 siRNA; shaded, cells transfected with Mcl-1 siRNA; black and white-dotted, cells transfected with Bcl-xL siRNA. siRNA against Bcl-xL significantly reduced the viability of LNCaP cells treated with $10 \mu \mathrm{M}$ of HS-1793 compared to siRNA against to Bcl-2 or Mcl-1 (** $\mathrm{P}<0.01)$. See Fig. 1 for other definitions.

(26). Cells become polyploid through a mechanism involving incomplete cell division (27). Although a proportion of hepatocytes are polyploid during normal development in mammals, a remarkable increase in the percentage of polyploid hepatocytes is observed in response to stress or injury (26). Moreover, heart muscle and vascular smooth-muscle cells, which are normally diploid, become polyploid in either rats or humans with hypertension $(27,28)$. However, it is not clear whether polyploidy is an adaptive or pathological response to these conditions.

It is believed that polyploidy plays a critical role in the development of genome instability and tumorigenesis (29). Polyploidy is often observed in cancer cells, but it is not clear 
whether this phenomenon is a cause or result of cancer development $(26,30,31)$. Previous studies showed that the failure of cytokinesis results in cell polyploidy and subsequent cell death (32) and that freshly generated polyploid cells are prone to undergo Bax-dependent mitochondrial membrane permeabilization and subsequent apoptosis (33). Polyploidization makes tumor cells more vulnerable to certain types of DNA damage, and increased polyploidy increases the sensitivity of cells to DNA-damaging agents (34). These findings suggest that, although polyploidy is involved in the development of neoplasia through genomic instability, it may also prove to be a new approach to cancer therapy. Conversely, polyploid cells may be resistant to DNA-damaging agents (33).

The present study elucidated that HS-1793 acts as an inducer of polyploidy. HS-1793 showed an exceptional ability to turn a non-synchronous population of proliferating cancer cells into a population with various degrees of polyploid cells (5 to $99 \%$ ) that is inversely proportional to Aurora B expression in multiple human cancer cells. Intriguingly, HS-1793 seems to exert polyploidy-inducing activity independent of Aurora B inhibition. Our Western blot assay showed that HS-1793 did not alter the expression level of Aurora B protein of the cancer cells we tested (negative data not shown). Furthermore, whereas HS-1793 efficiently induced polyploidy in Aurora B-depleted cells, it did not efficiently induce polyploidy in cells that express high levels of Aurora B. To date, several chemicals, including chemotherapeutic agents (22,35-37), have been shown to induce polyploidy in cancer cells. Among these, some agents can induce polyploidization independent of Aurora kinase inhibition, although most of these chemicals induce chromosome missegregation and cytokinesis failure through the loss of Aurora B, resulting in cell polyploidy. Thus, deciphering the detailed molecular mechanism by which HS-1793 induces polyploidization independent of Aurora B inhibition is an important and challenging task.

Intriguingly, the polyploidization induced by HS-1793 does not appear to cause definite commitment to apoptosis. Although approximately $100 \%$ of LNCaP cells displayed multinucleation $48 \mathrm{~h}$ after treatment with $10 \mu \mathrm{M}$ HS-1793, over $3 / 4$ of them were viable. Previous studies demonstrated that polyploidization is a genomic change that is thought to facilitate evolution through increased gene dosage, the capacity to overcome deleterious mutations, and increased genomic instability (38). This unexpected process infrequently generates viable progeny with normal fates, albeit far less frequently than diploid cells (39). Therefore, the induction of polyploidy in tumor cells may itself accelerate adaptation to and escape from chemotherapeutic agents, resulting in the evolution of more aggressive secondary tumors via genetic reshuffling. Thus, the termination of polyploid cells could provide an advantageous means to improve the efficacy of chemotherapeutic agents while minimizing the risk of genetically adapted tumor cells after chemotherapy.

Previous studies demonstrated that Bcl-xL/-2 supports viability during polyploidization $(22,24)$. A study showed that ectopic expression of Bcl-xL provides a survival advantage to polyploid murine FL5.12 cells after mitotic spindle damage (24). Another study showed that the combination of polyploidy inducers and agents targeting Bcl-xL exhibited synergistic antitumor activity. Further study of the mechanism by which Bcl-xL neutralization elicits cell death in polyploid cells showed that polyploidization shifts the burden to support viability from Mcl-1 to Bcl-xL (22). In the present study, we showed robust synergistic antitumor activity in LNCaP cells treated with HS-1793 in combination with ABT-263. Further, in line with previous hypotheses (22), siRNA-mediated knockdown of Bcl-xL but not Bcl-2 or Mcl-1 leads to enhanced polyploid-induced cell death by HS-1793. These data indicate that polyploidization in LNCaP cells induced by HS-1793 also may shift the burden to support viability to Bcl-xL. Down-regulation of Bcl-xL contributes to the vulnerability of tumor cells during polyploidization, suggesting that this may represent a new approach to preventing the evolution of secondary tumors that might be more prone to resist therapy. Thus, the elimination of polyploid cells can improve the efficacy of HS-1793 while minimizing the development of secondary tumors after chemotherapy. Combining HS-1793 with ABT-263 warrants further attention as a potential chemotherapeutic intervention in cancer cells that weakly express Aurora B.

Unlike HS-1793, resveratrol at the same dose does not induce multinucleation in LNCaP cells. In previous reports, the potency of stilbene analogs has been shown to depend on the chemical structure. Stilbene analogs with higher potency than resveratrol may have preferential interaction with the receptor (6). In addition, modulation of the activity of several genes or kinases or preferential subcellular localization due to the chemical configuration of the stilbene analogs may cause potent apoptosis-inducing activity $(40,41)$. HS-1793 does not contain the unstable double bond that is found in resveratrol, and the position of two of three hydroxyl groups in HS-1793 at the aromatic ring is different from resveratrol. Thus, although we assume that these configurations of HS-1793 might confer poly-ploidization activity, the structure-function effects of HS-1793 remain to be clarified.

In summary, we demonstrated here that the resveratrol analogue HS-1793 induces polyploidy in cancer cells that weakly express Aurora B. Further, polyploidization induced by HS-1793 is vulnerable to the downregulation of Bcl-xL.

\section{Acknowledgments}

This study was supported by a grant from the Korea Healthcare Technology R\&D Project, Ministry of Health and Welfare, Republic of Korea (A090314). The authors declare that they have no conflicts of interest.

\section{References}

1. Jang M, Cai L, Udeani GO, Slowing KV, Thomas CF, Beecher CW Fong HH, Farnsworth NR, Kinghorn AD, Mehta RG, Moon RC and Pezzuto JM: Cancer chemopreventive activity of resveratrol, a natural product derived from grapes. Science 275: 218-220, 1997.

2. Park JW, Choi YJ, Suh SI, Baek WK, Suh MH and Jin IN: Bcl-2 overexpression attenuates resveratrol-induced apoptosis in U937 cells by inhibition of caspase-3 activity. Carcinogenesis 22: 1633-1639, 2001.

3. Tinhofer I, Bernhard D, Senfter M, Anether G, Loeffler M, Kroemer G, Kofler R, Csordas A and Greil R: Resveratrol, a tumor-suppressive compound from grapes, induces apoptosis via a novel mitochondrial pathway controlled by Bcl-2. FASEB J 15: 1613-1615, 2001.

4. Trela BC and Waterhouse AL: Resveratrol: isomeric molar absorptivities and stability. J Agric Food Chem 44: 1253-1257, 1996. 
5. Song S, Lee H, Jin Y, Ha YM, Bae S and Chung HY: Syntheses of hydroxyl substituted 2-phenyl-naphthalenes as inhibitors of tyrosinase. Bioorg Med Chem Lett 17: 461-464, 2007.

6. Jeong SH, Jo WS, Song S, Suh H, Seol SY, Leem SH, Kwon TK and Yoo YH: A novel resveratrol derivative, HS1793, overcomes the resistance conferred by Bcl-2 in human leukemic U937 cells. Biochem Pharmacol 77: 1337-1347, 2009.

7. Jemal A, Siegel AR, Ward E, Hao Y, Xu J and Thun MJ: Cancer statistics. CA Cancer J Clin 59: 225-249, 2009.

8. Hsieh TC, Huang YC and Wu JM: Control of prostate cell growth, DNA damage and repair, and gene expression by resveratrol analogues, in vitro. Carcinogenesis 32: 93-101, 2011.

9. Lin HY, Shih A, Davis FB, Tang HY, Martino LJ, Bennett JA and Davis PJ: Resveratrol induced serine phosphorylation of p53 causes apoptosis in a mutant $\mathrm{p} 53$ prostate cancer cell line. J Urol 168: 748-755, 2002.

10. Wang Y, Romigh T, He X, Orloff MS, Silverman RH, Heston WD and Eng C: Resveratrol regulates the PTEN/AKT pathway through androgen receptor-dependent and -independent mechanisms in prostate cancer cell lines. Hum Mol Genet 19 4319-4329, 2010

11. Hsieh TC and Wu JM: Grape-derived chemopreventive agent resveratrol decreases prostate-specific antigen (PSA) expression in $\mathrm{LNCaP}$ cells by an androgen receptor (AR)-independent mechanism. Anticancer Res 20: 225-228, 2000.

12. Nigg EA: Mitotic kinases as regulators of cell division and its checkpoints. Nat Rev Mol Cell Biol 2: 21-32, 2001.

13. Carmena $\mathrm{M}$ and Earnshaw WC: The cellular geography of aurora kinases. Nat Rev Mol Cell Biol 4: 842-854, 2003.

14. Andrews PD, Knatko E, Moore WJ and Swedlow JR: Mitotic mechanics: the auroras come into view. Curr Opin Cell Biol 15: 672-683, 2003

15. Tseng TC, Chen SH, Hsu YP and Tang TK: Protein kinase profile of sperm and eggs: cloning and characterization of two novel testis-specific protein kinases (AIE1, AIE2) related to yeast and fly chromosome segregation regulators. DNA Cell Biol 17: 823-833, 1998.

16. Tatsuka M, Katayama H, Ota T, Tanaka T, Odashima S, Suzuki F and Terada Y: Multinuclearity and increased ploidy caused by overexpression of the aurora- and Ipl1-like midbody-associated protein mitotic kinase in human cancer cells. Cancer Res 58: 4811-4816, 1998

17. Chieffi P, Cozzolino L, Kisslinger A, Libertini S, Staibano S, Mansueto G, De Rosa G, Villacci A, Vitale M, Linardopoulos S, Portella G and Tramontano D: Aurora B expression directly correlates with prostate cancer malignancy and influence prostate cell proliferation. Prostate 66: 326-333, 2006.

18. Wilkinson RW, Odedra R, Heaton SP, Wedge SR, Keen NJ, Crafter C, Foster JR, Brady MC, Bigley A, Brown E, Byth KF, Barrass NC, Mundt KE, Foote KM, Heron NM, Jung FH, Mortlock AA, Boyle FT and Green S: AZD1152, a selective inhibitor of Aurora B kinase, inhibits human tumor xenograft growth by inducing apoptosis. Clin Cancer Res 13: 3682-3688, 2007.

19. Nair JS, de Stanchina E and Schwartz GK: The topoisomerase I poison CPT-11 enhances the effect of the Aurora B kinase inhibitor AZD1152 both in vitro and in vivo. Clin Cancer Res 15: 2022-2030, 2009.

20. Gizatullin F, Yao Y, Kung V, Harding MW, Loda M and Shapiro GI: The Aurora kinase inhibitor VX-680 induces endoreduplication and apoptosis preferentially in cells with compromised p53dependent postmitotic checkpoint function. Cancer Res 66: 7668-7677, 2006.

21. Lee EC, Frolov A, Li R, Ayala G and Greenberg NM: Targeting Aurora kinases for the treatment of prostate cancer. Cancer Res 66: 4996-5002, 2006

22. Shah OJ, Lin X, Li L, Huang X, Li J, Anderson MG, Tang H, Rodriguez LE, Warder SE, McLoughlin S, Chen J, Palma J, Glaser KB, Donawho CK, Fesik SW and Shen Y: Bcl-xL respresents a druggabel molecular vulnerability during aurora $\mathrm{B}$ inhibitor-mediated polyploidization. Proc Natl Acad Sci USA 107: 12634-12639, 2010.
23. Waizenegger I, Giménez-Abián JF, Wernic D and Peters JM: Regulation of human separase by securing binding and autocleavage. Curr Biol 12: 1368-1378, 2002.

24. Minn AJ, Boise LH and Thompson CB: Expression of Bcl-xL and loss of p53 can cooperate to overcome a cell cycle checkpoint induced by mitotic spindle damage. Genes Dev 10: 2621-2631, 1996.

25. Lanni JS and Jacks T: Characterization of the p53-dependent postmitotic checkpoint following spindle disruption. Mol Cell Biol 18: 1055-1064, 1998.

26. Andreassen PR, Lohez OD, Lacroix FB and Margolis RL: Tetraploid state induces p53-dependent arrest of nontransformed mammalian cells in G1. Mol Biol Cell 12: 1315-1328, 2001.

27. Guidotti JE, Brégerie O, Robert A, Debey P, Brechot C and Desdouets C: Liver cell polyploidization: a pivotal role for binuclear hepatocytes. J Biol Chem 278: 19095-19101, 2003.

28. Hixon ML, Obejero-Paz C, Muro-Cacho C, Wagner MW, Millie E, Nagy J, Hassold TJ and Gualberto A: Cks1 mediates vascular smooth muscle cell polyploidization, J Biol Chem 275 : 40434-40442, 2000

29. Matzke MA, Mette MF, Kanno T and Matzke AJ: Does the intrinsic instability of aneuploid genomes have a causal role in cancer? Trends Genet 19: 253-256, 2003.

30. Ganem NJ and Pellman D: Limiting the proliferation of polyploid cells. Cell 131: 437-440, 2007.

31. Ricke RM, van Ree JH and van Deursen JM: Whole chromosome instability and cancer: A complex relationship. Trends Genet 24: 457-466, 2008

32. Terada Y, Tatsuka M, Suzuki F, Yasuda Y, Fujita S and Otsu M: AIM-1: a mammalian midbody-associated protein required for cytokinesis. EMBO J 17: 667-676, 1998.

33. Castedo M, Coquelle A, Vivet S, Vitale I, Kauffmann A, Dessen P, Pequignot MO, Casares N, Valent A, Mouhamad S, Schmitt E, Modjtahedi N, Vainchenker W, Zitvogel L, Lazar V, Garrido C and Kroemer G: Apoptosis regulation in tetraploid cancer cells. EMBO J 25: 2584-2595, 2006.

34. Hau PM, Siu WY, Wong N, Lai PB and Poon RY: Polyploidization increases the sensitivity to DNA-damaging agents in mammalian cells. FEBS Lett 580: 4727-4736, 2006.

35. Xu WS, Perez G, Ngo L, Gui CY and Marks PA: Induction of polyploidy by histone deacetylase inhibitor: a pathway for antitumor effects. Cancer Res 65: 7832-7839, 2005.

36. Hall LL, Th'ng JP, Guo XW, Teplitz RL and Bradbury EM: A brief staurosporine treatment of mitotic cells triggers premature exit from mitosis and polyploid cell formation. Cancer Res 56: 3551-3559, 1996.

37. Reiners JJ Jr, Kleinman M, Joiakim A and Mathieu PA: The chemotherapeutic agents XK469 (2-\{4-[(7-chloro-2-quinoxalinyl) oxy]phenoxy\}propionic acid) and SH80 (2-\{4-[(7-bromo-2quinolinyl)oxy]phenoxy\}propionic acid) inhibit cytokinesis and promote polyploidy and induce senescence. J Pharmacol Exp Ther 328: 796-806, 2009.

38. Otto SP: The evolutionary consequences of polyploidy. Cell 131: 452-462, 2007.

39. Rancati G, Pavelka N, Fleharty B, Noll A, Trimble R, Walton K, Perera A, Staehling-Hampton K, Seidel CW and Li R: Aneuploidy underlies rapid adaptive evolution of yeast cells deprived of a conserved cytokinesis motor. Cell 135: 879-893, 2008.

40. Lee KW, Kang NJ, Rogozin EA, Oh S, Heo YS and Pugliese A: The resveratrol analogue 3,5,30,40,50-pentahydroxy-transstilbene inhibits cell transformation via MEK. Int J Cancer 123: 2487-2496, 2008

41. Gosslau A, Pabbaraja S, Knapp S and Chen KY: Trans- and cis-stilbene polyphenols induced rapid perinuclear mitochondrial clustering and $\mathrm{p} 53$-independent apoptosis in cancer cells but not normal cells. Eur J Pharm 587: 25-34, 2008. 